\title{
Rigorous Analysis of Internal Resonances in 3D Hybrid FE-BIE Formulations by means of the Poincaré-Steklov Operator
}

\author{
Freek Boeykens, Hendrik Rogier, Senior Member, IEEE, Jan Van Hese, Jeannick Sercu and Tim Boonen
}

\begin{abstract}
D hybrid finite element (FE) - boundary integral equation (BIE) formulations are widely used because of their ability to simulate large inhomogeneous structures in both open and bounded simulation domains by applying each method where it is the most efficient. However, some formulations suffer from breakdown frequencies at which the solution is not uniquely defined and errors are introduced due to internal resonances. In this paper, we investigate the occurrence of spurious solutions resulting from these resonances by using the concept of the Poincaré-Steklov or Dirichlet-to-Neumann operator, which provides a relation between the tangential electric field and the electric current on the boundary of a domain. By identifying this operator in both the FE and the BIE method, several new properties of internal resonances in 3D hybrid FE-BIE formulations are easily derived. Several conformal and nonconformal formulations are studied and the theory is then applied to a scattering problem.
\end{abstract}

Index Terms-hybrid FE-BIE, internal resonances, PoincaréSteklov, Dirichlet-to-Neumann

\section{INTRODUCTION}

$\mathbf{T}$ HE Finite Element (FE) method offers the ability to model complex inhomogeneous materials and anisotropic structures, but suffers from domain truncation and approximate absorbing boundary conditions. Its effectiveness can be improved by combining the technique with the Boundary Integral Equation (BIE) method, which allows extending the simulation domain to the full open space by enforcing the Silver-Müller radiation conditions in the kernel functions of the integral equations. Such methods were already successfully implemented in the past in 2D (e.g. [1], [2]) and in 3D (e.g. [3], [4]).

The exact hybrid formulation appears to be very important in order to avoid so-called spurious solutions. Previous contributions demonstrated that formulations applying the electric field integral equation (EFIE) or the magnetic field integral equation (MFIE) as BIE method in combination with an FE method contain certain 'forbidden frequencies' if the background medium is lossless [3], [5]-[12]. At these frequencies, the EFIE and MFIE are not uniquely defined and the sourceless hybrid system contains non-trivial solutions that introduce errors on the result. Adding some losses to the background

F. Boeykens and H. Rogier are with the Department of Information Technology, Ghent University, St. Pietersnieuwstraat 41, 9000 Ghent, Belgium. E-mail: freek.boeykens@intec.ugent.be.

J. Van Hese, J. Sercu and T. Boonen are with Agilent Technologies Belgium N.V., Kortrijksesteenweg 1093 B, 9051 Sint-Denijs-Westrem, Belgium. medium alleviates this problem, but doesn't provide much theoretical insight [13].

Some knowledge about spurious solutions is rendered by Chew in [14], [15], where he discusses internal resonances in integral equations by means of Gedanken experiments. $\mathrm{He}$ also relates the combined field integral equation (CFIE) to the cavity resonance problem with impedance boundary conditions to conclude that uniqueness is provided. The CFIE is also applied in time-domain FE-BIE methods, such as in [16], and in FDTD transparent boundary conditions, see [17], in order to overcome the problem of internal resonances. However, the stability of hybrid formulations such as in [4], [18], [19] was until now only investigated by means of numerical simulations, which is actually a trial-and-error method. Hence, another approach is still required.

In this paper, we build further on the approach applied in [20], where the concept of a Poincaré-Steklov (PS) or Dirichlet-to-Neumann $(\mathrm{DtN})$ operator is used to describe the relationship between the tangential electric field and the electric current on the boundary of a domain. By identifying this operator in the FE and BIE methods, different properties regarding internal resonances in hybrid formulations are easily derived. This also gives us the opportunity to investigate hybrid formulations on an operator level. Furthermore, we will not only investigate conformal formulations, where the FE and BIE share the same mesh, but also non-conformal formulations, where the FE and BIE solutions exist on different meshes and the continuity of the tangential electric fields and the electric currents is imposed in a weak sense.

The organisation of this paper is as follows. Section II presents the theoretical background for both the FE and BIE formulations. In Section III, some conformal hybrid formulations are studied and Section IV discusses non-conformal formulations. The results are shown in Section $\mathrm{V}$ and the conclusions are drawn in Section VI.

\section{General Formulations}

Consider the configuration of Fig. 1. A plane wave is scattered by an inhomogeneous domain $\Omega$, bounded by $\partial \Omega$, and with relative permittivity and permeability tensors $\overline{\bar{\epsilon}}_{r}(\mathbf{r})$ and $\overline{\bar{\mu}}_{r}(\mathbf{r})$, respectively. The domain is considered to consist of reciprocal material, hence the permittivity and permeability tensors are complex symmetrical, i.e. $\overline{\bar{\epsilon}}_{r}(\mathbf{r})=\overline{\bar{\epsilon}}_{r}^{T}(\mathbf{r})$ and $\overline{\bar{\mu}}_{r}(\mathbf{r})=\overline{\bar{\mu}}_{r}^{T}(\mathbf{r})$. The background medium is homogeneous and isotropic, characterised by $\epsilon_{0}$ and $\mu_{0}$. At the boundary $\partial \Omega$, 


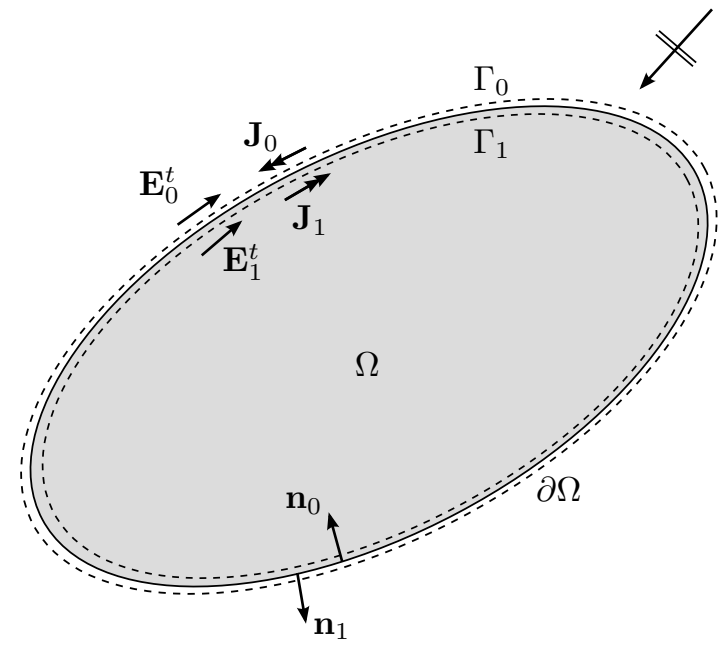

Fig. 1. Configuration of the scattering problem.

we define the surface $\Gamma_{0}$ as the smallest surface that encloses $\partial \Omega$ and $\Gamma_{1}$ as the largest surface that can be enclosed by $\partial \Omega$. Their outward normals are $\mathbf{n}_{0}$ and $\mathbf{n}_{1}$, respectively.

The tangential electric field on $\Gamma_{0}$ is $\mathbf{E}_{0}^{t}$ and the equivalent electric current on $\Gamma_{0}$ is $\mathbf{J}_{0}$. Accordingly, the tangential electric field on $\Gamma_{1}$ is $\mathbf{E}_{1}^{t}$ and the equivalent electric current on $\Gamma_{1}$ is $\mathbf{J}_{1}$. The relation between $\mathbf{E}_{0}^{t}$ and $\mathbf{J}_{0}$ is found using the BIE method and the connection between $\mathbf{E}_{1}^{t}$ and $\mathbf{J}_{1}$ is obtained by the FE method. This section describes these two formulations more in detail and identifies the complex symmetric PS operator $\mathcal{Y}=\mathcal{Y}^{\prime}$ that maps the tangential electric field on the equivalent electric current: $\mathcal{Y} \mathbf{E}^{t}=\mathbf{J}$ [21]. It is important to note that the transpose operator $\mathcal{Y}^{\prime}$ is defined with respect to the bilinear inner product $\langle\mathbf{X} \mid \mathbf{Y}\rangle=\int_{\partial \Omega} \mathbf{X} \cdot \mathbf{Y} \mathrm{d} S$ [22]. The frequencies for which $\mathcal{Y} \mathbf{E}^{t}=0$ are known as the Neumann eigenfrequencies and the frequencies for which $\mathcal{Y}^{-1} \mathbf{J}=0$ are the Dirichlet eigenfrequencies of the domain $\Omega$. All fields and sources are time-harmonic with a time dependency $e^{j \omega t}$.

\section{A. Finite Element Method}

In the inhomogeneous domain $\Omega$, the electric field is described by the wave equation

$$
\nabla \times \overline{\bar{\mu}}_{r}^{-1} \cdot\left(\nabla \times \mathbf{E}_{1}\right)-k_{0}^{2} \overline{\bar{\epsilon}}_{r} \cdot \mathbf{E}_{1}=0,
$$

with $k_{0}=\omega \sqrt{\epsilon_{0} \mu_{0}}$. The variational formulation is found after testing with weighting functions $\mathbf{W}_{1} \in \mathrm{H}(\operatorname{curl} ; \Omega)$ :

$$
\begin{array}{r}
\int_{\Omega}\left(\nabla \times \mathbf{W}_{1}\right) \cdot \overline{\bar{\mu}}_{r}^{-1} \cdot\left(\nabla \times \mathbf{E}_{1}\right) \mathrm{d} V-k_{0}^{2} \int_{\Omega} \mathbf{W}_{1} \cdot \overline{\bar{\epsilon}}_{r} \cdot \mathbf{E}_{1} \mathrm{~d} V \\
=j \omega \mu_{0} \int_{\Gamma_{1}} \mathbf{w}_{1} \cdot \mathbf{J}_{1} \mathrm{~d} S, \quad(2)
\end{array}
$$

where $\mathbf{w}_{1} \in \mathrm{H}^{-\frac{1}{2}}$ (curl, $\left.\Gamma_{1}\right)$ is the trace of $\mathbf{W}_{1}$ on $\Gamma_{1}$. Now, since only the boundary FE unknowns are required in order to couple with the BIE method, all interior unknowns are eliminated by reducing the LHS of (2) to its Schur complement $\mathcal{S}$. In this way, a direct relation between $\mathbf{E}_{1}^{t}$ and $\mathbf{J}_{1}$ is obtained. In operator notation, this becomes

$$
\mathcal{S} \mathbf{E}_{1}^{t}=j k_{0} \eta_{0} \mathbf{J}_{1}
$$

with $\eta_{0}=\sqrt{\frac{\mu_{0}}{\epsilon_{0}}}$. Note that at some frequencies the Schur complement $\mathcal{S}$ can become singular, meaning that its inverse $\mathcal{S}^{-1}$ is undefined. Therefore, at these frequencies, we implicitly extend $\Gamma_{1}$ with some interior edges, such that $\mathcal{S}^{-1}$ always exists. This is the equivalent of leaving some unknowns uneliminated while forming the Schur complement.

It is easily observed that $\mathcal{S}$ is a scaled version of the complex symmetric PS operator $\mathcal{Y}_{1}$, since it already provides a direct relationship between $\mathbf{E}_{1}^{t}$ and $\mathbf{J}_{1}$. The reduced $\mathrm{FE}$ system can thus be written as

$$
\mathcal{Y}_{1} \mathbf{E}_{1}^{t}=\mathbf{J}_{1}
$$

In the rest of this paper, we will refer to (4) as the Electric Field Formulation (EFF), as in [20].

Remark that, in the case of lossless reciprocal media, $\overline{\bar{\epsilon}}_{r}$ and $\overline{\bar{\mu}}_{r}$ are real symmetric tensors. For these materials, the LHS of (2) becomes a real symmetric matrix in a Galerkin weighting scheme (assuming the basis functions are realvalued). $\mathcal{S}$ is then a real symmetric matrix and $\mathcal{Y}_{1}$ becomes a purely imaginary operator that can be written as $\mathcal{Y}_{1}=j \mathcal{X}_{1}$, with $\mathcal{X}_{1}$ a real symmetric operator: $\mathcal{X}_{1}=\mathcal{X}_{1}^{\prime}$.

\section{B. Boundary Integral Equations}

The fields in the homogeneous background medium are related to $\mathbf{E}_{0}^{t}$ and $\mathbf{J}_{0}$ by integral equations with the Green's function $G_{0}\left(\mathbf{r}, \mathbf{r}^{\prime}\right)=e^{-j k_{0}\left|\mathbf{r}-\mathbf{r}^{\prime}\right|} /\left(4 \pi\left|\mathbf{r}-\mathbf{r}^{\prime}\right|\right)$ as integral kernel. These integral equations are defined by means of the following operators [23]:

$$
\begin{aligned}
& \mathcal{T}[\mathbf{X}](\mathbf{r})=- \frac{j \omega}{c} \mathbf{n} \times \int_{\Gamma_{0}} G_{0}\left(\mathbf{r}, \mathbf{r}^{\prime}\right) \mathbf{X}\left(\mathbf{r}^{\prime}\right) \mathrm{d} S^{\prime} \\
&+\frac{c}{j \omega} \mathbf{n} \times f_{\Gamma_{0}} \nabla G_{0}\left(\mathbf{r}, \mathbf{r}^{\prime}\right) \nabla^{\prime} \cdot \mathbf{X}\left(\mathbf{r}^{\prime}\right) \mathrm{d} S^{\prime}, \\
& \mathcal{K}[\mathbf{X}](\mathbf{r})=-\mathbf{n} \times \int_{\Gamma_{0}} \nabla G_{0}\left(\mathbf{r}, \mathbf{r}^{\prime}\right) \times \mathbf{X}\left(\mathbf{r}^{\prime}\right) \mathrm{d} S^{\prime},
\end{aligned}
$$

$\forall \mathbf{r} \in \Gamma_{0}$. The dashed integral symbol in (5a) denotes that the integration has to be computed in the Cauchy principal value sense. Using these operators, the block-Calderón operator is defined as

$$
\mathcal{P} \equiv\left(\begin{array}{cc}
-\mathcal{K}+\frac{1}{2} \mathcal{I} & -\eta_{0} \mathcal{T} \\
\frac{1}{\eta_{0}} \mathcal{T} & -\mathcal{K}+\frac{1}{2} \mathcal{I}
\end{array}\right),
$$

with $\mathcal{I}$ the identity operator. This block-Calderón operator provides the relations between the tangential electric field $\mathbf{E}^{t}$ and the equivalent electric current $\mathbf{J}$ in a domain where a normal $\mathbf{n}$ points into:

$$
\left(\begin{array}{c}
-\mathbf{n} \times \mathbf{E}^{t} \\
\mathbf{J}
\end{array}\right)=\mathcal{P}\left(\begin{array}{c}
-\mathbf{n} \times \mathbf{E}^{t} \\
\mathbf{J}
\end{array}\right) .
$$

In the configuration of Fig. 1, we solve for the tangential electric field $\mathbf{E}_{0}^{t}$ and the equivalent electric current $\mathbf{J}_{0}$ in the exterior domain. The normal $\mathbf{n}_{0}$ points to the outside of this domain, meaning that the complementary Calderón operator $\tilde{\mathcal{P}}=\mathcal{I}-\mathcal{P}$ needs to be used. In a system with sources, this 
becomes

$$
\begin{aligned}
(\mathcal{I}-\tilde{\mathcal{P}})\left(\begin{array}{c}
-\mathbf{n}_{0} \times \mathbf{E}_{0} \\
\mathbf{J}_{0}
\end{array}\right) & =\left(\begin{array}{c}
-\mathbf{n}_{0} \times \mathbf{E}^{i} \\
\mathbf{J}^{i}
\end{array}\right), \\
\left(\begin{array}{cc}
\mathcal{K}^{\prime}+\frac{1}{2} \mathcal{I} & \eta_{0} \mathcal{U} \\
-\frac{1}{\eta_{0}} \mathcal{N} \mathcal{U} \mathcal{N} & -\mathcal{K}+\frac{1}{2} \mathcal{I}
\end{array}\right)\left(\begin{array}{c}
\mathbf{E}_{0}^{t} \\
\mathbf{J}_{0}
\end{array}\right) & =\left(\begin{array}{c}
\mathbf{E}^{t, i} \\
\mathbf{J}^{i}
\end{array}\right) .
\end{aligned}
$$

We have that $\mathcal{N}=\mathbf{n}_{0} \times$, with $\mathcal{N}^{\prime}=-\mathcal{N}$ and $\mathcal{N}^{2}=-\mathcal{I}$. The complex symmetric operator $\mathcal{U}=\mathcal{U}^{\prime}$ is related to (5a) and (6) by $\mathcal{T}=\mathcal{N} \mathcal{U}$ and $\mathcal{N} \mathcal{K N}=\mathcal{K}^{\prime}$ [21]. The equations of (8b) are the well-known EFIE and MFIE.

The PS operator $\mathcal{Y}_{0}$, which relates $\mathbf{E}_{0}^{t}$ and $\mathbf{J}_{0}$ in the interior domain, can now be identified in the sourceless Stratton-Chu representation (7). Remark that the domain is supposed to be filled with background material $\left(\epsilon_{0}, \mu_{0}\right)$, since $G_{0}\left(\mathbf{r}, \mathbf{r}^{\prime}\right)$ is used as kernel in the integral equations. We obtain

$$
\frac{1}{2}\left(\begin{array}{c}
\mathbf{E}_{0}^{t} \\
\eta_{0} \mathbf{J}_{0}
\end{array}\right)=\left(\begin{array}{cc}
\mathcal{K}^{\prime} & \mathcal{U} \\
-\mathcal{N} \mathcal{U N} & -\mathcal{K}
\end{array}\right)\left(\begin{array}{c}
\mathbf{E}_{0}^{t} \\
\eta_{0} \mathbf{J}_{0}
\end{array}\right),
$$

and with $\mathcal{Y}_{0} \mathbf{E}_{0}^{t}=\mathbf{J}_{0}$, the following properties are found:

$$
\begin{aligned}
\left(-\mathcal{K}^{\prime}+\frac{1}{2} \mathcal{I}\right) \mathcal{Y}_{0}^{-1} & =\eta_{0} \mathcal{U} \\
\left(\mathcal{K}+\frac{1}{2} \mathcal{I}\right) \mathcal{Y}_{0} & =-\frac{1}{\eta_{0}} \mathcal{N} \mathcal{U N} .
\end{aligned}
$$

Similarly, the complementary PS operator $\tilde{\mathcal{Y}}_{0}$, which relates $\mathbf{E}_{0}^{t}$ and $\mathbf{J}_{0}$ in the exterior domain, can be identified in the sourceless EFIE and MFIE (8b):

$$
\frac{1}{2}\left(\begin{array}{c}
\mathbf{E}_{0}^{t} \\
\eta_{0} \mathbf{J}_{0}
\end{array}\right)=\left(\begin{array}{cc}
-\mathcal{K}^{\prime} & -\mathcal{U} \\
\mathcal{N} \mathcal{U N} & \mathcal{K}
\end{array}\right)\left(\begin{array}{c}
\mathbf{E}_{0}^{t} \\
\eta_{0} \mathbf{J}_{0}
\end{array}\right)
$$

and with $\tilde{\mathcal{Y}}_{0} \mathbf{E}_{0}^{t}=\mathbf{J}_{0}$, we obtain

$$
\begin{aligned}
\mathcal{K}^{\prime}+\frac{1}{2} \mathcal{I} & =-\eta_{0} \mathcal{U} \tilde{\mathcal{Y}}_{0}, \\
-\mathcal{K}+\frac{1}{2} \mathcal{I} & =\frac{1}{\eta_{0}} \mathcal{N} \mathcal{U N} \tilde{\mathcal{Y}}_{0}^{-1} .
\end{aligned}
$$

More details about $\mathcal{Y}_{0}$ and $\tilde{\mathcal{Y}}_{0}$ can be found in [21].

The uniqueness of the EFIE and the MFIE solutions is easily established after identification of the PS operator $\tilde{\mathcal{Y}}_{0}$ in $(8 b)$. For the sourceless EFIE we find with (12a):

$$
\eta_{0} \mathcal{U}\left(\tilde{\mathcal{Y}}_{0} \mathbf{E}_{0}^{t}-\mathbf{J}_{0}\right)=0
$$

which indicates that spurious solutions can exist when $\mathcal{U}$ becomes singular. From (10a), it is clear that this occurs at the Dirichlet eigenfrequencies of the interior structure filled with background material. Hence, at these frequencies, a nonzero spurious electric current $\mathbf{J}_{\mathrm{sp}}$ can be found. Remark that $\mathbf{J}_{\text {sp }}$ does not radiate, since it is a resonant current on the PEC cavity formed by $\Omega$. It will only generate a non-zero field inside the cavity. However, it will induce a radiating spurious tangential electric field $\mathbf{E}_{\mathrm{sp}}^{t}=\tilde{\mathcal{Y}}_{0}^{-1} \mathbf{J}_{\mathrm{sp}}$.

For the sourceless MFIE, we obtain using (12b):

$$
\frac{1}{\eta_{0}} \mathcal{N} \mathcal{U N}\left(\mathbf{E}_{0}^{t}-\tilde{\mathcal{Y}}_{0}^{-1} \mathbf{J}_{0}\right)=0
$$

which shows that now spurious solutions can exist when $\mathcal{N} \mathcal{U N}$ becomes singular. From (10b), we find that this occurs at the Neumann eigenfrequencies of the interior structure filled with background material. Hence, at these frequencies, a nonzero spurious tangential electric field $\mathbf{E}_{\mathrm{sp}}^{t}$ can be found. This is a resonant tangential electric field on the PMC cavity formed by $\Omega$, which will only generate a non-zero field inside the cavity. However, it will induce a radiating spurious electric current $\mathbf{J}_{\mathrm{sp}}=\tilde{\mathcal{Y}}_{0} \mathbf{E}_{\mathrm{sp}}^{t}$.

It is important to note that the spurious solutions of (13) and (14) do not coincide [23]. Therefore, no spurious solutions can exist if both equations are satisfied.

\section{CONFORMAL Hybrid FE/BIE FORMULATIONS}

A first group of hybrid formulations consists of conformal formulations. In these systems, both the FE and BIE method are applied on the same mesh and the tangential electric fields and equivalent electric currents are coupled via strong (pointwise) continuity relations

$$
\begin{aligned}
\mathbf{E}_{0}^{t} & =\mathbf{E}_{1}^{t}, \\
\mathbf{J}_{0} & =-\mathbf{J}_{1} .
\end{aligned}
$$

The solution of these systems is, however, not always uniquely defined and the frequencies for which internal resonances occur can be predicted by identifying the PS operator in the different formulations.

\section{A. EFF and EFIE}

The classic approach to construct a hybrid FE-BIE formulation is to combine the EFF and the EFIE [3], [8]. Making use of (4) and the first equation of (8b), the following system is obtained:

$$
\left(\begin{array}{cc}
\mathcal{Y}_{1} & \mathcal{I} \\
\mathcal{K}^{\prime}+\frac{1}{2} \mathcal{I} & \eta_{0} \mathcal{U}
\end{array}\right)\left(\begin{array}{c}
\mathbf{E}_{0}^{t} \\
\mathbf{J}_{0}
\end{array}\right)=\left(\begin{array}{c}
0 \\
\mathbf{E}^{t, i}
\end{array}\right) .
$$

It is well-known that this formulation suffers from internal resonances, more precisely at the Dirichlet eigenfrequencies of the interior structure filled with background medium [15]. This occurs because of the non-uniqueness of the EFIE for the background medium. In order to verify this, the PS operator $\tilde{\mathcal{Y}}_{0}$ is first identified in the sourceless EFIE, as in (13), and after substitution of the first equation into this expression, we find for the electric current:

$$
\eta_{0} \mathcal{U}\left(\tilde{\mathcal{Y}}_{0} \mathcal{Y}_{1}^{-1}+\mathcal{I}\right) \mathbf{J}_{0}=0
$$

This leads to $\mathbf{J}_{0}=0$, unless $\eta_{0} \mathcal{U}$ or $\left(\tilde{\mathcal{Y}}_{0} \mathcal{Y}_{1}^{-1}+\mathcal{I}\right)$ becomes singular. In Appendix A it is shown that the singularity of the latter leads to physical resonances, so spurious resonances are only present when $\eta_{0} \mathcal{U}$ is singular. As already demonstrated in Section II-B, this occurs at the Dirichlet eigenfrequencies of the interior structure filled with background material. At these frequencies we thus find a non-radiating resonant spurious electric current $\mathbf{J}_{\mathrm{sp}}$ and a radiating induced spurious tangential electric field $\mathbf{E}_{\mathrm{sp}}^{t}=-\mathcal{Y}_{1}^{-1} \mathbf{J}_{\mathrm{sp}}$. 


\section{B. EFF and MFIE}

Another approach is to combine the EFF and the MFIE. Now (4) and the second equation of (8b) are utilised to form the following system:

$$
\left(\begin{array}{cc}
\mathcal{Y}_{1} & \mathcal{I} \\
-\frac{1}{\eta_{0}} \mathcal{N} \mathcal{U N} & -\mathcal{K}+\frac{1}{2} \mathcal{I}
\end{array}\right)\left(\begin{array}{c}
\mathbf{E}_{0}^{t} \\
\mathbf{J}_{0}
\end{array}\right)=\left(\begin{array}{c}
0 \\
\mathbf{J}^{i}
\end{array}\right)
$$

This formulation also suffers from internal resonances, but now at the Neumann eigenfrequencies of the interior structure filled with background medium, due to the non-uniqueness of the MFIE. This is again verified after identification of $\tilde{\mathcal{Y}}_{0}$ in the homogeneous matrix system. Making use of (14), and after substitution of the first equation into this expression, we find:

$$
\frac{1}{\eta_{0}} \mathcal{N} \mathcal{U N}\left(\mathcal{I}+\tilde{\mathcal{Y}}_{0}^{-1} \mathcal{Y}_{1}\right) \mathbf{E}_{0}^{t}=0
$$

This leads to $\mathbf{E}_{0}^{t}=0$, unless $\frac{1}{\eta_{0}} \mathcal{N} \mathcal{U N}$ or $\left(\mathcal{I}+\tilde{\mathcal{Y}}_{0}^{-1} \mathcal{Y}_{1}\right)$ is singular. Again, it is shown in Appendix A that the singularity of $\left(\mathcal{I}+\tilde{\mathcal{Y}}_{0}^{-1} \mathcal{Y}_{1}\right)$ leads to physical resonances, which means that spurious solutions can only occur when $\frac{1}{\eta_{0}} \mathcal{N} \mathcal{U N}$ is singular. It is shown in Section II-B that this occurs at the Neumann eigenfrequencies of the interior structure filled with background material. At these frequencies we thus find a nonradiating spurious tangential electric field $\mathbf{E}_{\mathrm{sp}}$ and a radiating induced spurious electric current $\mathbf{J}_{\mathrm{sp}}=-\mathcal{Y}_{1} \mathbf{E}_{\mathrm{sp}}^{t}$.

Remark that these spurious solutions differ from the spurious solutions in Section III-A, since in (19), they occur due to the non-uniqueness of the MFIE, whereas the spurious solutions in (17) exist because of the non-uniqueness of the EFIE.

\section{EFF and CFIE}

A solution to the problem in previous formulations is to employ the CFIE in the BIE domain [3]. The system matrix then becomes a linear combination of the system matrices in III-A and III-B. We get

$$
\begin{aligned}
\left\{\alpha\left(\begin{array}{cc}
\mathcal{Y}_{1} & \mathcal{I} \\
\mathcal{K}^{\prime}+\frac{1}{2} & \eta_{0} \mathcal{U}
\end{array}\right)+\right. & \left.\left(\begin{array}{cc}
\mathcal{Y}_{1} & \mathcal{I} \\
-\frac{1}{\eta_{0}} \mathcal{N} \mathcal{U} \mathcal{N} & -\mathcal{K}+\frac{1}{2}
\end{array}\right)\right\}\left(\begin{array}{c}
\mathbf{E}_{0}^{t} \\
\mathbf{J}_{0}
\end{array}\right) \\
& =\left(\begin{array}{c}
0 \\
\alpha-\alpha \mathbf{E}^{t, i}+(1-\alpha) \mathbf{J}^{i}
\end{array}\right),
\end{aligned}
$$

with $0<\alpha<1$. Using (12), the sourceless matrix system becomes

$$
\begin{aligned}
& \left\{\alpha\left(\begin{array}{cc}
\mathcal{Y}_{1} & \mathcal{I} \\
-\eta_{0} \mathcal{U} \tilde{\mathcal{Y}}_{0} & \eta_{0} \mathcal{U}
\end{array}\right)+\right. \\
& \left.(1-\alpha)\left(\begin{array}{cc}
\mathcal{Y}_{1} & \mathcal{I} \\
\left(\mathcal{K}-\frac{1}{2}\right) \tilde{\mathcal{Y}}_{0} & -\mathcal{K}+\frac{1}{2}
\end{array}\right)\right\}\left(\begin{array}{l}
\mathbf{E}_{0}^{t} \\
\mathbf{J}_{0}
\end{array}\right)=\left(\begin{array}{l}
0 \\
0
\end{array}\right) .
\end{aligned}
$$

First of all, note that the spurious solutions of Section III-A and Section III-B can never be solutions of this system, since they are different from each other, as explained in the previous section and in [23]. Therefore, at the Dirichlet and Neumann eigenfrequencies of the interior structure filled with background medium, the solution of (21) will be zero. Possible spurious solutions for the electric current can be found by substituting the first equation of (21) into the second one. We obtain:

$$
\left(\alpha \eta_{0} \mathcal{U}+(1-\alpha)\left(-\mathcal{K}+\frac{1}{2}\right)\right)\left(\tilde{\mathcal{Y}}_{0} \mathcal{Y}_{1}^{-1}+\mathcal{I}\right) \mathbf{J}_{0}=0
$$

With the transposed equation of (10a), this is further simplified to

$$
\left(\alpha+(1-\alpha) \mathcal{Y}_{0}\right) \eta_{0} \mathcal{U}\left(\tilde{\mathcal{Y}}_{0} \mathcal{Y}_{1}^{-1}+\mathcal{I}\right) \mathbf{J}_{0}=0
$$

This leads to $\mathbf{J}_{0}=0$, unless $\left(\alpha+(1-\alpha) \mathcal{Y}_{0}\right)$ or $\eta_{0} \mathcal{U}$ or $\left(\tilde{\mathcal{Y}}_{0} \mathcal{Y}_{1}^{-1}+\mathcal{I}\right)$ is singular. We already know that the singularity of the latter corresponds with physical resonances and that the singularity of $\eta_{0} \mathcal{U}$ cannot lead to internal resonances since it occurs at the Dirichlet eigenfrequencies of the interior structure filled with background medium. Hence, it is clear that spurious solutions can only exist when $\left(\alpha+(1-\alpha) \mathcal{Y}_{0}\right)$ is singular. Therefore, $\frac{\alpha}{\alpha-1}$ has to be an eigenvalue of $\mathcal{Y}_{0}$ (or $\frac{\alpha-1}{\alpha}$ an eigenvalue of $\left.\mathcal{Y}_{0}^{-1}\right)$. This means that $\mathcal{Y}_{0}$ should have a real non-zero eigenvalue. However, for a lossless reciprocal medium, $\mathcal{Y}_{0}$ is a pure imaginary symmetric matrix, so it possesses only imaginary eigenvalues. Therefore, no internal resonances are present.

The proof for the tangential electric field is similar and leads to the same conclusions.

\section{D. $E F F+M F I E$ and $E F I E$}

The last conformal formulation under study adds up the EFF and the MFIE in one equation and combines it with the EFIE in order to form a symmetric system. The system is then [18], [19]:

$$
\left(\begin{array}{cc}
\mathcal{Y}_{1}+\frac{1}{\eta_{0}} \mathcal{N} \mathcal{U N} & \mathcal{K}+\frac{1}{2} \mathcal{I} \\
\mathcal{K}^{\prime}+\frac{1}{2} \mathcal{I} & \eta_{0} \mathcal{U}
\end{array}\right)\left(\begin{array}{l}
\mathbf{E}_{0}^{t} \\
\mathbf{J}_{0}
\end{array}\right)=\left(\begin{array}{c}
-\mathbf{J}^{i} \\
\mathbf{E}^{t, i}
\end{array}\right)
$$

Although one would expect that the solution of (24) is uniquely defined since both the EFIE and the MFIE are used to model the background medium, internal resonances are still present due to the construction of the hybrid system matrix. Indeed, after transposing (12a), we find that $\mathcal{K}+\frac{1}{2} \mathcal{I}$ and $\mathcal{U}$ have a shared nullspace:

$$
\mathcal{K}+\frac{1}{2} \mathcal{I}=-\eta_{0} \tilde{\mathcal{Y}}_{0} \mathcal{U}
$$

Therefore, spurious solutions of the form $\left(\begin{array}{lll}\mathbf{0} & \mathbf{J}_{\mathrm{sp}}\end{array}\right)^{T}$ can exist. From (10a), it is clear that this occurs at the Dirichlet eigenfrequencies of the interior structure filled with background material.

Remark that, in contrast to the internal resonances of Section III-A and Section III-B, no tangential electric field is induced here. 


\section{NON-CONFORMAL HYBRID FE/BIE FORMULATIONS}

A second group of hybrid formulations consists of nonconformal formulations. In these systems, the FE and BIE methods are independently discretised and the solutions are projected from one domain to the other. These projections can be calculated analytically and do not largely extend the simulation time [24]. A great advantage of these formulations is that higher order FE basis functions can be used in combination with first-order BIE basis functions. Also, domain decomposition techniques can be employed to solve the hybrid system.

Depending on the transmission conditions, different formulations exist. In this paper, we focus on weak tangential continuity and Robin transmission conditions.

\section{A. Tangential Continuity}

In this formulation, the transmission conditions are the continuity of the tangential electric and magnetic field at the boundary $\partial \Omega$ :

$$
\begin{aligned}
\mathbf{E}_{0}^{t} & =\mathbf{E}_{1}^{t}, \\
\mathbf{J}_{0} & =-\mathbf{J}_{1},
\end{aligned}
$$

which are applied in a weak sense. After combination with the EFF, EFIE and MFIE, the following symmetric system matrix is obtained:

$$
\left(\begin{array}{cccc}
\mathcal{Y}_{1} & -\frac{1}{2} \mathcal{I} & 0 & \frac{1}{2} \mathcal{I} \\
-\frac{1}{2} \mathcal{I} & 0 & \frac{1}{2} \mathcal{I} & 0 \\
0 & \frac{1}{2} \mathcal{I} & \frac{1}{\eta_{0}} \mathcal{N} \mathcal{U} \mathcal{N} & \mathcal{K} \\
\frac{1}{2} \mathcal{I} & 0 & \mathcal{K}^{\prime} & \eta_{0} \mathcal{U}
\end{array}\right)\left(\begin{array}{c}
\mathbf{E}_{1}^{t} \\
\mathbf{J}_{1} \\
\mathbf{E}_{0}^{t} \\
\mathbf{J}_{0}
\end{array}\right)=\left(\begin{array}{c}
0 \\
0 \\
-\mathbf{J}^{i} \\
\mathbf{E}^{t, i}
\end{array}\right)
$$

It is clear that the continuity of the tangential electric field is explicitly enforced in the weak sense (in the second equation), whereas the continuity of the electric current is only implicitly defined. The investigation of internal resonances starts from the sourceless matrix representation and after elimination of $\mathbf{E}_{1}^{t}$ we find:

$$
\left(\begin{array}{ccc}
-\frac{1}{2} \mathcal{I} & \mathcal{Y}_{1} & \frac{1}{2} \mathcal{I} \\
\frac{1}{2} \mathcal{I} & \frac{1}{\eta_{0}} \mathcal{N} \mathcal{U} \mathcal{N} & \mathcal{K} \\
0 & \mathcal{K}^{\prime}+\frac{1}{2} \mathcal{I} & \eta_{0} \mathcal{U}
\end{array}\right)\left(\begin{array}{c}
\mathbf{J}_{1} \\
\mathbf{E}_{0}^{t} \\
\mathbf{J}_{0}
\end{array}\right)=\left(\begin{array}{l}
0 \\
0 \\
0
\end{array}\right)
$$

The electric current $\mathbf{J}_{1}$ can also be eliminated from the first equation and we obtain:

$$
\left(\begin{array}{cc}
\mathcal{Y}_{1}+\frac{1}{\eta_{0}} \mathcal{N} \mathcal{U N} & \mathcal{K}+\frac{1}{2} \mathcal{I} \\
\mathcal{K}^{\prime}+\frac{1}{2} \mathcal{I} & \eta_{0} \mathcal{U}
\end{array}\right)\left(\begin{array}{l}
\mathbf{E}_{0}^{t} \\
\mathbf{J}_{0}
\end{array}\right)=\left(\begin{array}{l}
0 \\
0
\end{array}\right)
$$

This is the same system matrix as (24), so the same spurious solutions will exist. This occurs at the Dirichlet eigenfrequencies of the interior structure filled with background medium.

\section{B. Robin Transmission Conditions}

The Robin transmission conditions find their origin in the Silver-Müller radiation condition that states that the energy radiated from sources cannot scatter back from infinity:

$$
\lim _{r \rightarrow \infty} r\left(\mathbf{u}_{r} \times(\nabla \times \mathbf{E})-j k \mathbf{E}\right)=0 .
$$

At the boundary $\partial \Omega$, a similar residue can be calculated:

$$
\psi=-(\mathbf{n} \times(\nabla \times \mathbf{E})-j k \mathbf{E})=\mathbf{E}^{t}+\eta_{0} \mathbf{J} .
$$

This residue is then transferred to the other domain:

$$
\begin{aligned}
& \mathbf{E}_{0}^{t}+\eta_{0} \mathbf{J}_{0}=\mathbf{E}_{1}^{t}-\eta_{0} \mathbf{J}_{1}, \\
& \mathbf{E}_{1}^{t}+\eta_{0} \mathbf{J}_{1}=\mathbf{E}_{0}^{t}-\eta_{0} \mathbf{J}_{0},
\end{aligned}
$$

In order to formulate the boundary conditions, the constant $\eta_{0}$ is replaced by a general real number $\alpha$ and we obtain:

$$
\begin{aligned}
& \mathbf{E}_{0}^{t}+\alpha \mathbf{J}_{0}=\mathbf{E}_{1}^{t}-\alpha \mathbf{J}_{1}, \\
& \mathbf{E}_{1}^{t}+\alpha \mathbf{J}_{1}=\mathbf{E}_{0}^{t}-\alpha \mathbf{J}_{0} .
\end{aligned}
$$

After combining the EFF, EFIE and MFIE, the following symmetric matrix representation is obtained [4]:

$$
\begin{aligned}
& \left\{\begin{array}{cccc}
\mathcal{Y}_{1} & -\frac{1}{2} \mathcal{I} & 0 & \frac{1}{2} \mathcal{I} \\
-\frac{1}{2} \mathcal{I} & 0 & \frac{1}{2} \mathcal{I} & 0 \\
0 & \frac{1}{2} \mathcal{I} & \frac{1}{\eta_{0}} \mathcal{N} \mathcal{U} \mathcal{N} & \mathcal{K} \\
\frac{1}{2} \mathcal{I} & 0 & \mathcal{K}^{\prime} & \eta_{0} \mathcal{U}
\end{array}\right) \\
& \left.+\left(\begin{array}{cccc}
\frac{1}{2 \alpha} \mathcal{I} & 0 & -\frac{1}{2 \alpha} \mathcal{I} & 0 \\
0 & -\frac{1}{2} \alpha \mathcal{I} & 0 & -\frac{1}{2} \alpha \mathcal{I} \\
-\frac{1}{2 \alpha} \mathcal{I} & 0 & \frac{1}{2 \alpha} \mathcal{I} & 0 \\
0 & -\frac{1}{2} \alpha \mathcal{I} & 0 & -\frac{1}{2} \alpha \mathcal{I}
\end{array}\right)\right\}\left(\begin{array}{c}
\mathbf{E}_{1}^{t} \\
\mathbf{J}_{1} \\
\mathbf{E}_{0}^{t} \\
\mathbf{J}_{0}
\end{array}\right) \\
& =\left(\begin{array}{c}
0 \\
0 \\
-\mathbf{J}^{i} \\
\mathbf{E}^{t, i}
\end{array}\right) \text {. }
\end{aligned}
$$

Again, in order to find internal resonances, we start from the sourceless matrix representation and eliminate $\mathbf{E}_{1}^{t}$.

$$
\left(\begin{array}{ccc}
-\alpha \mathcal{Y}_{1}-\mathcal{I} & \mathcal{Y}_{1} & -\alpha \mathcal{Y}_{1} \\
\mathcal{I} & \frac{1}{\eta_{0}} \mathcal{N} \mathcal{U} \mathcal{N} & \mathcal{K}+\frac{1}{2} \mathcal{I} \\
-\alpha \mathcal{I} & \mathcal{K}^{\prime}+\frac{1}{2} \mathcal{I} & \eta_{0} \mathcal{U}-\alpha
\end{array}\right)\left(\begin{array}{l}
\mathbf{J}_{1} \\
\mathbf{E}_{0}^{t} \\
\mathbf{J}_{0}
\end{array}\right)=\left(\begin{array}{l}
0 \\
0 \\
0
\end{array}\right)
$$

The second and third equation can be replaced by the following linear combinations:

$$
\left\{\begin{array}{l}
-\eta_{0}\left(\mathcal{K}+\frac{1}{2} \mathcal{I}\right)(\text { eq. } 2)+\mathcal{N} \mathcal{U N}(\text { eq. } 3) \\
-\eta_{0} \mathcal{U}(\text { eq. } 2)+\left(\mathcal{K}^{\prime}-\frac{1}{2} \mathcal{I}\right)(\text { eq. } 3)
\end{array}\right.
$$

By applying the Calderón identities (see Appendix B), this is simplified to

$$
\begin{cases}\left(\eta_{0}\left(\mathcal{K}+\frac{1}{2} \mathcal{I}\right)+\alpha \mathcal{N} \mathcal{U N}\right)\left(\mathbf{J}_{0}+\mathbf{J}_{1}\right) & =0 \\ \left(\eta_{0} \mathcal{U}+\alpha\left(\mathcal{K}^{\prime}-\frac{1}{2} \mathcal{I}\right)\right)\left(\mathbf{J}_{0}+\mathbf{J}_{1}\right) & =0\end{cases}
$$

and after identifying the PS operator $\mathcal{Y}_{0}$ by means of (10), we find:

$$
\begin{cases}\mathcal{N} \mathcal{U N}\left(\mathcal{Y}_{0}^{-1}-\alpha \mathcal{I}\right)\left(\mathbf{J}_{1}+\mathbf{J}_{0}\right) & =0 \\ \left(\mathcal{K}^{\prime}-\frac{1}{2}\right)\left(\mathcal{Y}_{0}^{-1}-\alpha \mathcal{I}\right)\left(\mathbf{J}_{1}+\mathbf{J}_{0}\right) & =0\end{cases}
$$

Hence, $\mathbf{J}_{0}=-\mathbf{J}_{1}$, unless a shared nullspace is found between $\mathcal{N U N}\left(\mathcal{Y}_{0}^{-1}-\alpha \mathcal{I}\right)$ and $\left(\mathcal{K}^{\prime}-\frac{1}{2}\right)\left(\mathcal{Y}_{0}^{-1}-\alpha \mathcal{I}\right)$. Therefore, $\alpha$ should be an eigenvalue of $\mathcal{Y}_{0}^{-1}$ (or $\frac{1}{\alpha}$ an eigenvalue of $\mathcal{Y}_{0}$ ). The same reasoning as for the CFIE can be used here to 


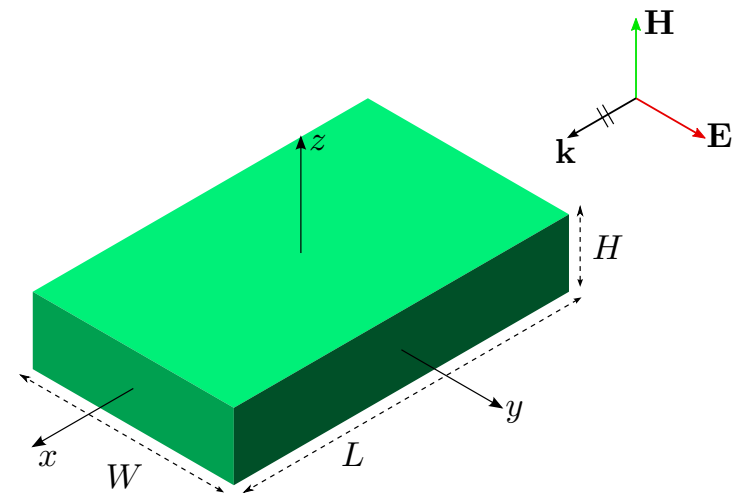

Fig. 2. Configuration of the simulated problem. A plane wave is scattered by a dielectric cuboid with relative permittivity $\epsilon_{r}=2$.

conclude that $\mathcal{Y}_{0}^{-1}$ only has imaginary non-zero eigenvalues if the background medium is lossless and reciprocal. So, for real $\alpha \neq\{0, \infty\}$, (35) becomes:

$$
\left(\begin{array}{cc}
\mathcal{Y}_{1} & \mathcal{I} \\
\frac{1}{\eta_{0}} \mathcal{N} \mathcal{U} \mathcal{N} & \mathcal{K}-\frac{1}{2} \mathcal{I} \\
\mathcal{K}^{\prime}+\frac{1}{2} \mathcal{I} & \eta_{0} \mathcal{U}
\end{array}\right)\left(\begin{array}{l}
\mathbf{E}_{0}^{t} \\
\mathbf{J}_{0}
\end{array}\right)=\left(\begin{array}{l}
0 \\
0 \\
0
\end{array}\right) .
$$

Here, both the EFIE and MFIE are solved for the background medium, so the solution will always be uniquely defined. Hence this formulation is free of internal resonances.

\section{Numerical Results}

In this Section, the above concepts are illustrated by means of numerical simulations. The configuration of the simulated problem is depicted in Fig. 2. A plane wave, travelling along the positive $x$-axis and with the electric field polarised along the positive $y$-axis, is scattered by a dielectric cuboid with dimensions $\{L, W, H\}$ and relative permittivity $\epsilon_{r}=2$. As expansion functions, the curl conforming first-order edge elements $\mathbf{w}_{i}$ [25] are used for $\mathbf{E}_{i}^{t}$ and the divergence conforming RWGs $\mathbf{v}_{i}$ [26] are used for $\mathbf{J}_{i}$. We have that $\mathbf{w}_{i}=\mathbf{n}_{i} \times \mathbf{v}_{i}$. Galerkin weighting is applied in all formulations.

Remark that the Dirichlet and Neumann eigenfrequencies are equal for 3D configurations, because of the duality principle. In order to prove this, we use the example of Fig. 3. The eigenfrequencies of the Dirichlet problem in 3(a) are equal to the eigenfrequencies of Neumann the problem in 3(b) because the configurations are dual to each other. This is also true for the problems 3(c) and 3(d) (the terms Dirichlet and Neumann refer to the electric field solution). Now, since the eigenfrequencies are found from

$$
\nabla \times \nabla \times \mathbf{E}-k^{2} \mathbf{E}=0,
$$

it is clear that the eigensolutions are only frequency dependent through the wavenumber

$$
k=\frac{2 \pi f \sqrt{\epsilon_{r} \mu_{r}}}{c_{0}} .
$$

This implies that the eigenfrequencies of Figs. 3(a) and 3(c) are also equal, because the wave number for both configurations is the same. Hence, the eigenfrequencies of 3(a) and

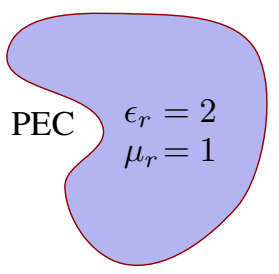

(a)

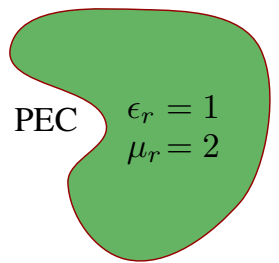

(c)

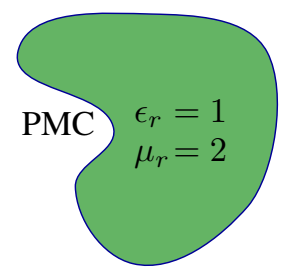

(b)

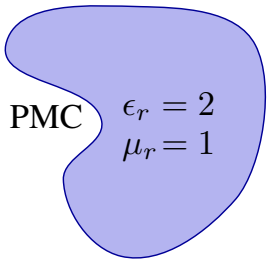

(d)
Fig. 3. Example of the duality principle. Configuration (a) is dual to configuration (b) and configuration (c) is dual to configuration (d).

3(b) are equal. The latter two problems are the Dirichlet and Neumann problem of the same configuration, respectively. Hence, the Dirichlet and Neumann eigenfrequencies are equal.

\section{A. Discretised PS operator}

In (3), it was already shown that $\mathcal{Y}_{1}$ can be calculated by rescaling the Schur complement of the FE system matrix with $j k_{0} \eta_{0}$. If a Green's function kernel is available, such as for homogeneous regions $\Omega$, the same operator can also be computed via the BIE method and the obtained matrices must be the same up to discretisation errors.

In order to obtain the BIE PS operator $\mathcal{Y}_{1}$, we expand the tangential electric field and the equivalent electric current in first-order edge element and RWG basis functions, respectively:

$$
\begin{aligned}
\mathbf{E}^{t} & =\sum_{n=1}^{N} \chi_{n} \mathbf{w}_{n}, \\
\mathbf{J} & =\sum_{n=1}^{N} \xi_{n} \mathbf{v}_{n} .
\end{aligned}
$$

The discretised PS operator then becomes the complex symmetric $N \times N$ matrix $\mathrm{Y}$, with

$$
\mathrm{Y}_{i j}=\left\langle\mathbf{w}_{i} \mid \mathcal{Y} \mathbf{w}_{j}\right\rangle .
$$

Here, both test and basis functions are the curl conforming first order edge elements $\mathbf{w}_{i}$, since $\mathcal{Y}_{1}$ operates on the tangential field $\mathbf{E}^{t}$ and results in an equivalent electric current that needs to be tested with functions from its dual space.

After inserting (42) into the sourceless Stratton-Chu formulation (9) and into the definition of the PS operator $\left(\mathcal{Y} \mathbf{E}^{t}=\mathbf{J}\right)$, we obtain [21]

$$
\begin{aligned}
\frac{1}{2} \mathrm{D}^{T} \chi & =\mathrm{A}^{T} \chi+\eta_{1} \mathrm{~B} \xi, \\
\frac{1}{2} \eta_{1} \mathrm{D} \xi & =-\mathrm{C} \chi-\eta_{1} \mathrm{~A} \xi, \\
\mathrm{Y} \chi & =\mathrm{D} \xi,
\end{aligned}
$$




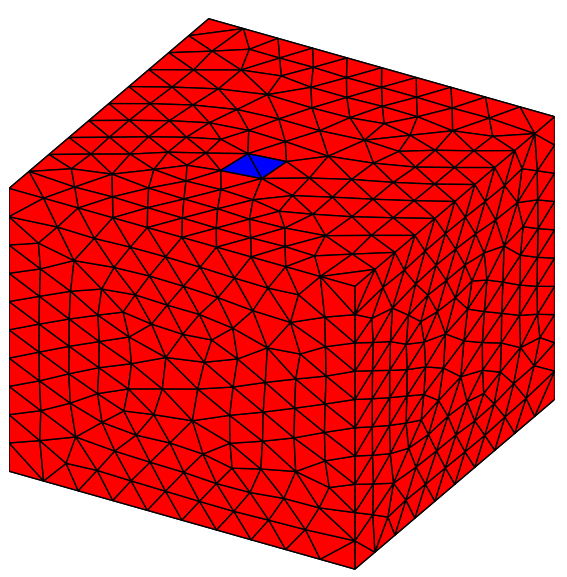

Fig. 4. Mesh used to calculate the FE and BIE PS operator $\mathcal{Y}_{1}$. The interactions of the marked RWG with the other basis functions are displayed in Fig. 5.

with $\chi$ and $\xi$ the vectors containing the unknowns $\chi_{n}$ and $\xi_{n}$, and the $N \times N$ matrices $\mathrm{A}, \mathrm{B}, \mathrm{C}$ and $\mathrm{D}$ defined as

$$
\begin{aligned}
\mathrm{A}_{i j} & =\left\langle\mathbf{w}_{i} \mid \mathcal{K} \mathbf{v}_{j}\right\rangle, \\
\mathrm{B}_{i j} & =\left\langle\mathbf{v}_{i} \mid \mathcal{U} \mathbf{v}_{j}\right\rangle, \\
\mathrm{C}_{i j} & =\left\langle\mathbf{w}_{i} \mid \mathcal{N} \mathcal{U} \mathcal{N} \mathbf{w}_{j}\right\rangle, \\
\mathrm{D}_{i j} & =\left\langle\mathbf{w}_{i} \mid \mathbf{v}_{j}\right\rangle .
\end{aligned}
$$

Note that the calculation is performed for the inner domain, so the Calderón operator $\mathcal{P}$ needs to be employed. Also, in order to obtain $\mathcal{Y}_{1}$, the Green's function $G_{1}\left(\mathbf{r}, \mathbf{r}^{\prime}\right)$ is used (with $\epsilon_{r}=$ 2 ). Now, since the matrices $B$ and $C$ are complex symmetric, it is easily shown from (44) that the discretised PS operator can be written in an explicitly symmetric form as

$$
\mathrm{Y}=\frac{1}{\eta_{1}}\left(-\mathrm{C}+\left(\frac{1}{2} \mathrm{D}-\mathrm{A}\right)^{T} \mathrm{~B}^{-1}\left(\frac{1}{2} \mathrm{D}-\mathrm{A}\right)\right) .
$$

The matrices $Y_{\mathrm{FE}}$, obtained from (3), and $\mathrm{Y}_{\mathrm{BIE}}$, obtained from (46), are now compared against each other for the problem of Fig. 2 with $\{L, W, H\}=\{1,1,1\} \mathrm{m}$ at a frequency of $100 \mathrm{MHz}$. The matrix $\mathrm{Y}_{\mathrm{FE}}$ is calculated twice, once with first-order FE basis functions and a second time with secondorder FE basis functions. The mesh of the configuration is depicted in Fig. 4. It has an average edge length of $100 \mathrm{~mm}$ and leads to 2040 degrees of freedom on the boundary. Remark that, for the FE calculations, the interior mesh has the same average edge length as the boundary mesh. For the first-order FE computations, this leads to a total of 8086 unknowns and for the second-order FE computations, we have 41815 unknowns.

The numerical equivalence of the PS operators is illustrated in Fig. 5, where the imaginary part of the 512th column of $Y_{\mathrm{BIE}}$ and $Y_{F E}$ (obtained once via first-order and a second time via second-order FE basis functions) are compared. This column represents the interactions between the marked basis function in Fig. 4 and all other basis functions. In Fig. 5(a) the singular close interactions are shown and errors between the matrices are visible because only in the BIE method special routines

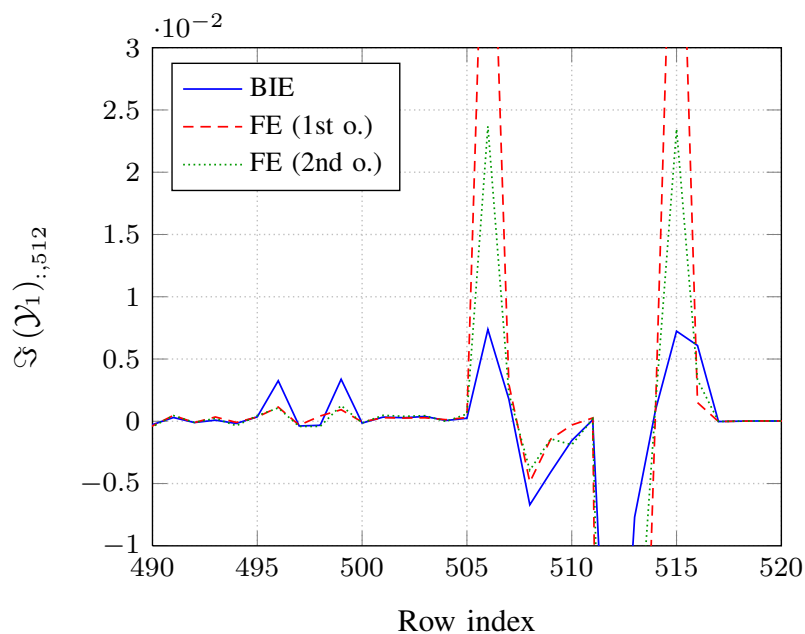

(a)

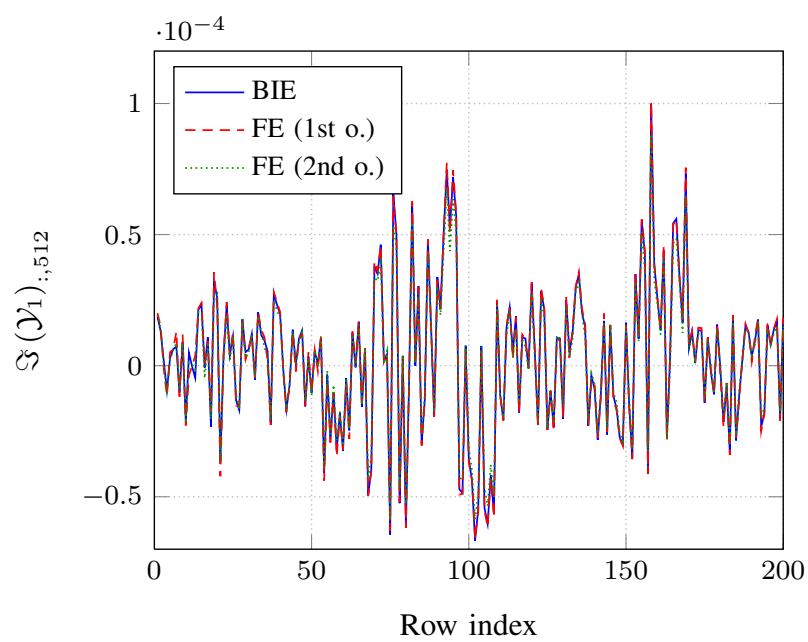

(b)

Fig. 5. Imaginary part of the 512th column of the discretised PS operators $\mathrm{Y}_{\mathrm{BIE}}$ and $\mathrm{Y}_{\mathrm{FE}}$, where the FE PS operator is calculated once with first-order basis functions and a second time with second-order basis functions. Some singular (a) and non-singular (b) interactions are shown.

were used to calculate these selfpatch integrals [27]. We also observed that this error became even worse when investigating the interactions between basis functions on the edges or in corners. However, it is seen that the accuracy of $Y_{\mathrm{FE}}$ increases by using second-order FE basis functions.

Some non-singular interactions are shown in Fig. 5(b) and here we observe a very good match between the PS operators. The same results are obtained for the other columns.

\section{B. Spurious Solutions}

In Section III and IV, it is shown that internal resonances can occur at the Dirichlet and Neumann eigenfrequencies of the FE domain filled with background medium for the formulations under study. Here, we will demonstrate this for the scattering problem of Fig. 2 with $\{L, W, H\}=$ $\{1.2,0.5,0.2\} \mathrm{m}$ using a mesh with an average edge length of $70 \mathrm{~mm}$. As mentioned before, the Dirichlet and Neumann eigenfrequencies are identical for $3 \mathrm{D}$ configurations and for a 
TABLE I

EIGENFREQUENCIES OF THE VACUUM AND DIELECTRIC CUBOID.

\begin{tabular}{ccc}
\hline mode $(l, m, n)$ & $f[\mathrm{MHz}]$ for $\epsilon_{r}=1$ & $f$ [MHz] for $\epsilon_{r}=2$ \\
\hline$(1,0,0)$ & 124.913524 & 88.327200 \\
$(2,0,0)$ & 249.827048 & 176.654400 \\
$(0,1,0)$ & 299.792458 & 211.985280 \\
$(1,1,0)$ & 324.775163 & 229.650720 \\
$(3,0,0)$ & 374.740572 & 264.981600 \\
$(2,1,0)$ & 390.242325 & 275.942994 \\
$(3,1,0)$ & 479.902089 & 339.342021 \\
\hline
\end{tabular}

box, they are found from

$$
f_{l, m, n}=\frac{c}{2 \sqrt{\epsilon_{r}}} \sqrt{\frac{l^{2}}{1.2^{2}}+\frac{m^{2}}{0.5^{2}}+\frac{n^{2}}{0.2^{2}}} .
$$

These frequencies are shown in Table I for both the background medium $\left(\epsilon_{r}=1\right)$ and the dielectric medium $\left(\epsilon_{r}=2\right)$. The numerical simulations are then performed as follows. A reference solution $X_{\text {PMCHWT }}$ is first calculated by means of the pure BIE Poggio-Miller-Chang-Harrington-Wu-Tsai (PMCHWT) formulation and then a combined error in $\mathbf{E}_{0}^{t}$ and $\mathbf{J}_{0}$ with respect to this solution is computed using the solution vectors $X$ for each of the formulations under study:

$$
\epsilon=\frac{\left\|X-X_{\text {PMCHWT }}\right\|}{\left\|X_{\text {PMCHWT }}\right\|} .
$$

The error for the conformal formulations is shown in Fig. 6(a) for a frequency range from $300 \mathrm{MHz}$ to $350 \mathrm{MHz}$ and the corresponding condition numbers of the hybrid system matrices are displayed in Fig. 6(b). As expected, all conformal formulations exhibit spurious solutions except the formulation of III-C, where the CFIE was computed with $\alpha=0.5$. The breakdown frequency of formulations III-A and III-D occurs exactly as predicted, whereas it has shifted towards lower frequencies for formulation III-B. This is because different integration routines were used to calculate the BIE interactions in formulation III-B (in a Galerkin scheme, these interactions form an $\mathbf{n} \times$ MFIE formulation). Moreover, the $\mathcal{K}$ operator is not well tested in this formulation, what leads to the broader error peak. A solution would be to employ Buffa-Christiansen test functions for the second equation [28], [29], but then there is no Galerkin testing anymore. Also note that with these test functions, the formulation still suffers from internal resonances, since these occur in the continuous problem, independent from any discretisation.

Observing Fig. 6(b), we see that the condition number increases for all formulations that have breakdown frequencies around $325 \mathrm{MHz}$. Other increases of the condition numbers are also observed at $337 \mathrm{MHz}$ and $346 \mathrm{MHz}$. These peaks are a bifurcation of the expected peak at $339 \mathrm{MHz}$, due to the singularity of $\mathcal{Y}_{1}$. Although, it is clear from Fig. 6(a) that this does not contribute to errors on the solution vector.

Fig. 7(a) compares the solution error defined by (48) for the non-conformal FE/BIE formulations discussed in Section IV. Since these formulations completely decouple the FE and BIE discretisations, it is also possible to compare the solutions from FE/BIE formulations with second-order FE basis functions. As

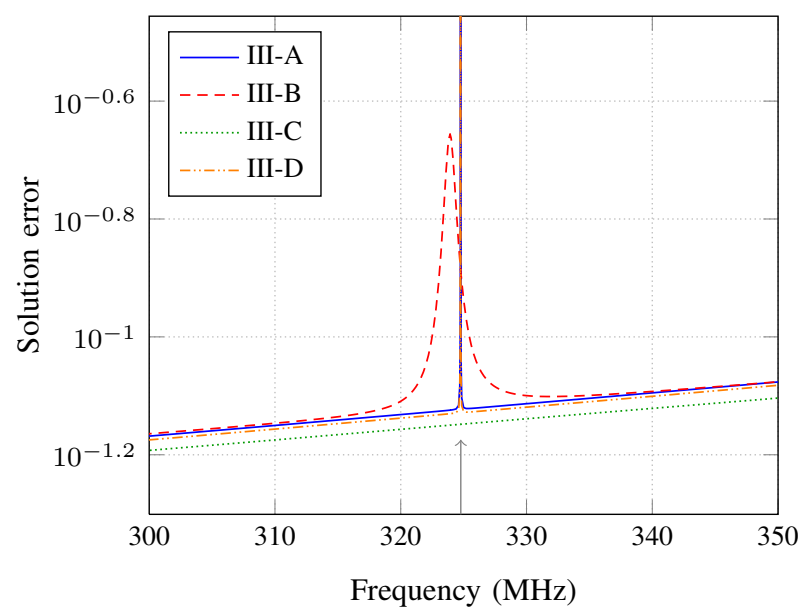

(a)

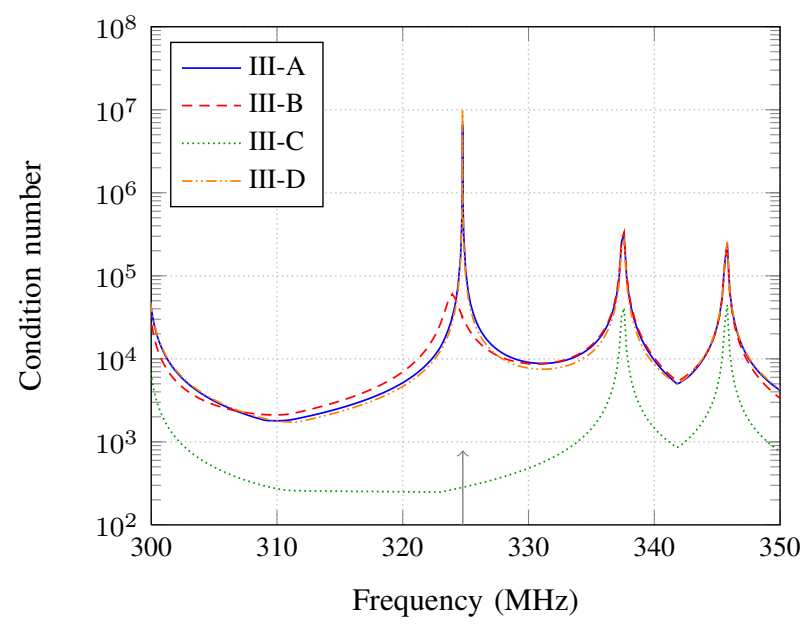

(b)

Fig. 6. Combined error of the solutions of the conformal formulations with respect to the reference PMCHWT solution (a) and the corresponding condition numbers of the hybrid system matrices (b). The theoretical breakdown frequency is shown by the vertical arrow.

explained in the previous Section, the formulation imposing weak tangential continuity of electric and magnetic fields suffers from internal resonances, whereas the Robin boundary conditions (with $\alpha=\eta_{0}$ ) lead to a resonance-free solution. A difference in accuracy between first and second-order FE basis functions is also noticeable.

The condition numbers of the system matrices of the nonconformal formulations are shown in Fig. 7(b). The resonance of $\mathcal{Y}_{1}$ can be noticed for these formulations too and the bifurcation has disappeared for the formulations with secondorder FE basis functions. Again, this resonance does not contribute to errors on the solution vector, as illustrated in Fig. 7(a).

\section{Radiating vs. Non-Radiating Spurious Solutions}

In Section III, it was already shown that not all spurious solutions radiate. The internal resonance solutions of the PEC or PMC cavity filled with background material produce a non-zero field in the cavity, but do not radiate outside $\Omega$, whereas the solutions that are induced by these resonance 


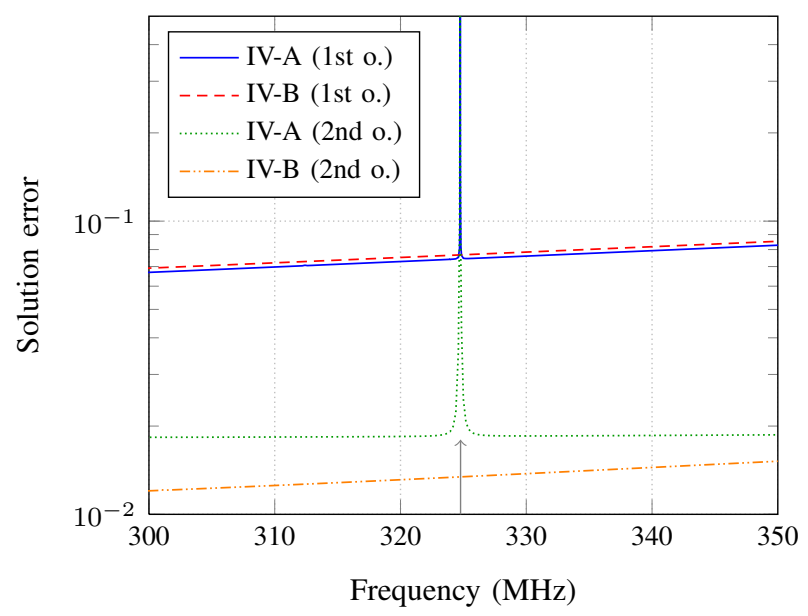

(a)

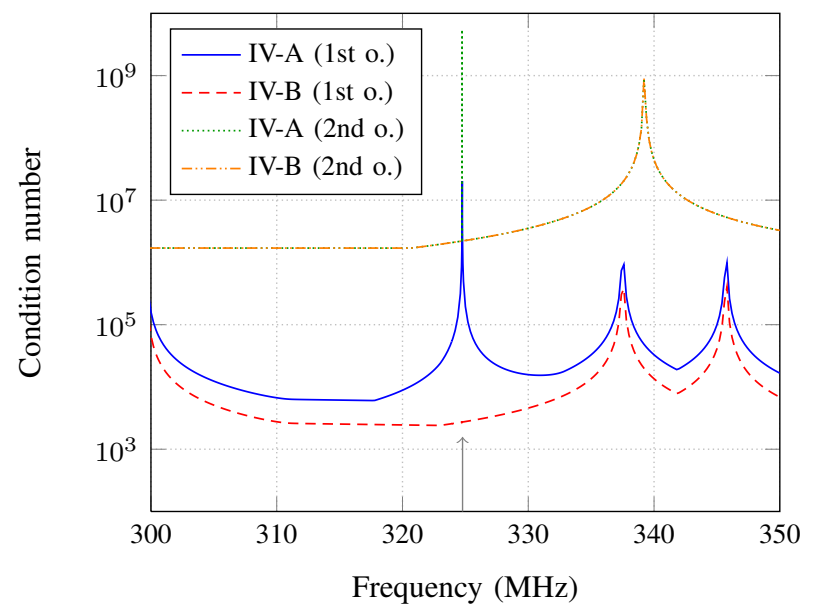

(b)

Fig. 7. Combined error of the solutions of the non-conformal formulations with respect to the reference PMCHWT solution (a) and the corresponding condition numbers of the hybrid system matrices (b). The theoretical breakdown frequency is shown by the vertical arrow.

currents or fields do radiate outside the cavity. Here, we will demonstrate this for the example of Fig. 2 with $\{L, W, H\}=$ $\{1.2,0.5,0.2\} \mathrm{m}$ using a mesh with an average edge length of $70 \mathrm{~mm}$. The normalised radar cross section (RCS) $\sigma / \lambda^{2}$ is first calculated in the $y z$-plane at a frequency of $324.75 \mathrm{MHz}$ by means of a reference PMCHWT formulation. This RCS is then compared with the RCS obtained from each of the spurious solutions of Section III and Section IV.

Fig. 8(a) displays the RCS in the $y z$-plane for the reference PMCHWT solution and for the spurious tangential electric fields $\mathbf{E}_{\mathrm{sp}}$ of the formulations in III-A and III-B. The spurious tangential electric field of formulation III-A is induced by a resonant current and one can observe that this produces a spurious RCS with the same order of magnitude as the correct reference RCS. The field radiated by the spurious tangential electric field of formulation III-B is clearly much lower in magnitude and it can be concluded that these spurious solutions do not radiate.

In Fig. 8(b), the RCS in the $y z$-plane is shown for the reference PMCHWT solution and for the spurious electric

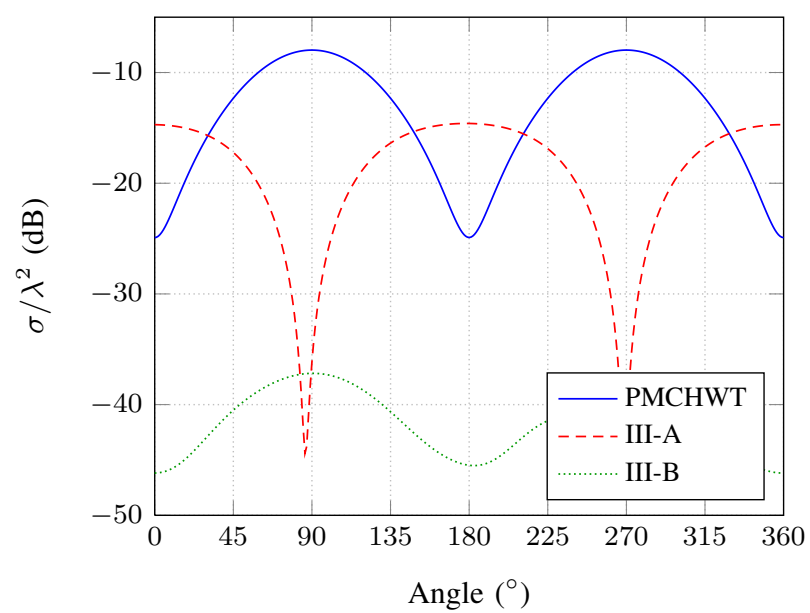

(a)

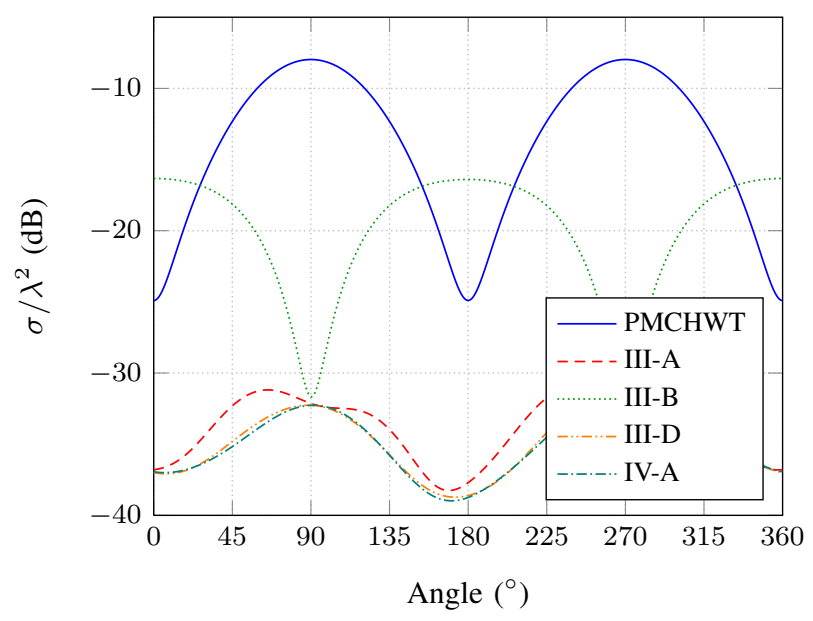

(b)

Fig. 8. RCS in the $y z$-plane for the spurious tangential electric fields (a) and the spurious electric currents (b). The RCS calculated from a reference PMCHWT solution is also displayed in order to compare the order of magnitude.

currents $\mathbf{J}_{\mathrm{sp}}$ of the formulations in III-A, III-B, III-D and IV-A. Again, it is clear that only the currents that are induced by a resonant tangential electric field radiate, whereas the internal resonant currents do not produce a field outside $\Omega$.

\section{CONCLUSION}

In this paper the stability of several conformal and nonconformal hybrid FE/BIE formulations was analysed by identifying the Poincaré-Steklov operator in the system matrices. This method identifies and explains all internal resonances and even predicts their frequencies for simple configurations.

All conformal formulations except the EFF/CFIE formulation exhibited spurious solutions at the Dirichlet or Neumann eigenfrequencies of the $\mathrm{FE}$ domain filled with background medium. We also proved that the EFF/CFIE system is free of internal resonances because the PS operator $\mathcal{Y}_{0}$ has no real eigenvalues.

The non-conformal formulations under study were the formulation with weak tangential continuity of electric and magnetic fields at the interface and the formulation with Robin 
boundary conditions. It was shown that tangential continuity leads to spurious solutions and that Robin boundary conditions are free from internal resonances as long as the parameter $\alpha$ is real and different from $\{0, \infty\}$.

The equivalence between the discretised FE and BIE PS operators was also demonstrated. However, a mismatch between the singular selfpatch interactions was found due to numerical issues. Furthermore, the theory was verified for a scattering problem and it was shown that only the induced resonance currents (or fields) radiate outside the cavity.

\section{ACKNOWLEDGMENT}

The work of F. Boeykens was supported by a doctoral grant from the Agency for Innovation by Science and Technology in Flanders (IWT).

\section{APPENDIX A \\ PHYSICAL RESONANCES}

When solving the sourceless problem of Fig. 1, the Maxwell equations lead to the following expressions in the exterior and interior domain:

$$
\left\{\begin{array}{l}
\tilde{\mathcal{Y}}_{0} \mathbf{E}_{0}^{t}-\mathbf{J}_{0}=0 \\
\mathcal{Y}_{1} \mathbf{E}_{1}^{t}-\mathbf{J}_{1}=0
\end{array}\right.
$$

In the case of conformal meshes, we have that $\mathbf{E}_{0}^{t}=\mathbf{E}_{1}^{t}$ and $\mathbf{J}_{0}=-\mathbf{J}_{1}$. After substituting this in (A-1) and pre-multiplying both equations with $\tilde{\mathcal{Y}}_{0}^{-1}$, we find

$$
\left(\mathcal{I}+\tilde{\mathcal{Y}}_{0}^{-1} \mathcal{Y}_{1}\right) \mathbf{E}_{0}^{t}=0
$$

This leads to $\mathbf{E}_{0}^{t}=0$, unless $\left(\mathcal{I}+\tilde{\mathcal{Y}}_{0}^{-1} \mathcal{Y}_{1}\right)$ becomes singular. In this case, we find a physical resonance tangential electric field for the configuration.

A similar expression for the electric current can be found, starting from

$$
\left\{\begin{array}{l}
\mathbf{E}_{0}^{t}-\tilde{\mathcal{Y}}_{0}^{-1} \mathbf{J}_{0}=0 \\
\mathbf{E}_{1}^{t}-\mathcal{Y}_{1}^{-1} \mathbf{J}_{1}=0
\end{array}\right.
$$

After substitution of $\mathbf{E}_{1}^{t}$ by $\mathbf{E}_{0}^{t}$ and $\mathbf{J}_{1}$ by $-\mathbf{J}_{1}$, and after pre-multiplication of (A-3) with $\tilde{\mathcal{Y}}_{0}$, we obtain

$$
\left(\tilde{\mathcal{Y}}_{0} \mathcal{Y}_{1}^{-1}+\mathcal{I}\right) \mathbf{J}_{0}=0
$$

This leads to $\mathbf{J}_{0}=0$, unless $\left(\tilde{\mathcal{Y}}_{0} \mathcal{Y}_{1}^{-1}+\mathcal{I}\right)$ becomes singular. In this case, we find a physical resonance electric current for the configuration.

\section{APPENDIX B}

\section{The CALderón IDENTities}

The sourceless Calderón operator (9) is written as

$$
\frac{1}{2}\left(\begin{array}{c}
\mathbf{E} \\
\eta \mathbf{J}
\end{array}\right)=\mathcal{H}\left(\begin{array}{c}
\mathbf{E} \\
\eta \mathbf{J}
\end{array}\right)
$$

Applying the Hamiltonian operator $\mathcal{H}$ [21] again on (B-1) leads to

$$
\begin{aligned}
\frac{1}{2} \mathcal{H}\left(\begin{array}{c}
\mathbf{E} \\
\eta \mathbf{J}
\end{array}\right) & =\mathcal{H}^{2}\left(\begin{array}{c}
\mathbf{E} \\
\eta \mathbf{J}
\end{array}\right), \\
\frac{1}{4}\left(\begin{array}{c}
\mathbf{E} \\
\eta \mathbf{J}
\end{array}\right) & =\mathcal{C}\left(\begin{array}{c}
\mathbf{E} \\
\eta \mathbf{J}
\end{array}\right),
\end{aligned}
$$

with

$$
\mathcal{C}=\left(\begin{array}{cc}
\mathcal{K}^{\prime 2}-\mathcal{U N} \mathcal{U N} & \mathcal{K}^{\prime} \mathcal{U}-\mathcal{U} \mathcal{K} \\
-\mathcal{N} \mathcal{U N} \mathcal{K}^{\prime}+\mathcal{K} \mathcal{N} \mathcal{U N} & -\mathcal{N} \mathcal{U N U}+\mathcal{K}^{2}
\end{array}\right)
$$

Since (B-3) has to be valid for all frequencies, 4 equations are found, the so-called Calderón identities.

\section{REFERENCES}

[1] T. Cwik, "Coupling finite element and integral equation solutions using decoupled boundary meshes," IEEE Trans. Antennas Propag., vol. 40, no. 12, pp. 1496-1504, Dec. 1992.

[2] D. J. Hoppe, L. W. Epp, and J.-F. Lee, "A hybrid symmetric FEM/MOM formulation applied to scattering by inhomogeneous bodies of revolution," IEEE Trans. Antennas Propag., vol. 42, no. 6, pp. 798-805, Apr. 1994.

[3] J. L. Volakis, K. Sertel, and B. C. Usner, Frequency Domain Hybrid Finite Element Methods for Electromagnetics, C. A. Balanis, Ed. Morgan \& Claypool, 2006.

[4] K. Zhao, M. N. Vouvakis, and J.-F. Lee, "Solving electromagnetic problems using a novel symmetric FEM-BEM approach," IEEE Trans. Magn., vol. 42, no. 4, pp. 583-586, Apr. 2006.

[5] E. Arvas and J. R. Mautz, "On the non-uniqueness of the surface EFIE applied to multiple conducting and/or dielectric bodies," Archiv für Elektronik und Übertragungstechnik (AË̈), vol. 42, no. 6, pp. 364 369, 1988.

[6] B.-S. Yang, A. W. Glisson, and P. M. Goggans, "Interior resonance problems associated with hybrid integral equation/partial differential equation methods," in Antennas and Propagation Society International Symposium, 1992. AP-S. 1992 Digest. Held in Conjuction with: URSI Radio Science Meeting and Nuclear EMP Meeting., IEEE, vol. 2, Jul. 1992, pp. 781-784.

[7] J.-J. Angélini, C. Soize, and P. Soudais, "Hybrid numerical method for harmonic 3-D Maxwell equations: scattering by a mixed conducting and inhomogeneous anisotropic dielectric medium," IEEE Trans. Antennas Propag., vol. 41, no. 1, pp. 66-76, Jan. 1993.

[8] X.-Q. Sheng, J.-M. Jin, J. Song, C.-C. Lu, and W. C. Chew, "On the formulation of hybrid finite-element and boundary-integral methods for 3-D scattering," IEEE Trans. Antennas Propag., vol. 46, no. 3, pp. 303311, Mar. 1998.

[9] Y. Ji, H. Wang, and T. H. Hubbing, "A numerical investigation of interior resonances in the hybrid FEM/MoM method," IEEE Trans. Antennas Propag., vol. 51, no. 2, pp. 347-349, Feb. 2003.

[10] M. Smith and A. Peterson, "Numerical solution of the CFIE using vector bases and dual interlocking meshes," IEEE Trans. Antennas Propag., vol. 53, no. 10, pp. 3334-3339, Oct. 2005.

[11] F. Andriulli and E. Michielssen, "A regularized combined field integral equation for scattering from 2-D perfect electrically conducting objects," IEEE Trans. Antennas Propag., vol. 55, no. 9, pp. 2522-2529, Sep. 2007.

[12] S. Yan, J.-M. Jin, and Z. Nie, "Calderón preconditioner: From EFIE and MFIE to N-Müller equations," IEEE Trans. Antennas Propag., vol. 58, no. 12, pp. 4105-4110, Dec. 2010.

[13] J. Collins, J.-M. Jin, and J. Volakis, "Eliminating interior resonances in finite element-boundary integral methods for scattering," IEEE Trans. Antennas Propag., vol. 40, no. 12, pp. 1583 -1585, Dec. 1992.

[14] W. C. Chew and J. M. Song, "Gedanken experiments to understand the internal resonance problems of electromagnetic scattering," Electromagnetics, vol. 27, no. 8, pp. 457-471, Nov. 2007.

[15] W. C. Chew, M. S. Tong, and B. Hu, Integral Equation Methods for Electromagnetic and Elastic Waves, C. A. Balanis, Ed. Morgan \& Claypool, 2008

[16] D. Jiao, A. Ergin, B. Shanker, E. Michielssen, and J.-M. Jin, "A fast higher-order time-domain finite element-boundary integral method for 3-D electromagnetic scattering analysis," IEEE Trans. Antennas Propag., vol. 50, no. 9, pp. 1192-1202, Sep. 2002. 
[17] M. Lu, B. Shanker, and E. Michielssen, "Elimination of spurious solutions associated with exact transparent boundary conditions in fdtd solvers," IEEE Antennas Wireless Propag. Lett., vol. 3, pp. 59-62, Dec. 2004.

[18] M. Vouvakis, S.-C. Lee, K. Zhao, and J.-F. Lee, "A symmetric FEM-IE formulation with a single-level IE-QR algorithm for solving electromagnetic radiation and scattering problems," IEEE Trans. Antennas Propag., vol. 52, no. 11, pp. 3060 - 3070, Nov. 2004.

[19] M. M. Botha and J.-M. Jin, "On the variational formulation of hybrid finite element-boundary integral techniques for electromagnetic analysis," IEEE Trans. Antennas Propag., vol. 52, no. 11, pp. 3037-3047, Nov. 2004.

[20] P. Demarcke and H. Rogier, "The Poincaré-Steklov operator in hybrid Finite Element-Boundary Integral Equation formulations," IEEE Antennas Wireless Propag. Lett., vol. 10, pp. 503-506, May 2011.

[21] L. Knockaert and D. De Zutter, "On the complex symmetry of the Poincaré-Steklov operator," Progress In Electromagnetics Research B, vol. 7, pp. 145-157, 2008.

[22] S. R. Garcia and M. Putinar, "Complex symmetric operators and applications," Transactions of the American Mathematical Society, vol. 358, no. 3, pp. 1285-1315, May 2005.

[23] J. G. Van Bladel, Electromagnetic Fields, 2nd ed., D. G. Dudley, Ed. John Wiley and Sons, Inc., 2007.

[24] F. Boeykens, H. Rogier, J. Van Hese, J. Sercu, and T. Boonen, "Efficient calculation of coupling matrices for a decoupled FE/BIE formulation," in Electromagnetics in Advanced Applications (ICEAA), 2012 International Conference on, 2012, pp. $506-509$.

[25] J.-C. Nédélec, "Mixed finite elements in R3," Numerische Mathematik, vol. 35, pp. 315-341, 1980.

[26] S. M. Rao, D. R. Wilton, and A. W. Glisson, "Electromagnetic scattering by surfaces of arbitrary shapes," IEEE Trans. Antennas Propag., vol. 30, no. 3, pp. 409-418, May 1982.

[27] J. Peeters, I. Bogaert, and D. De Zutter, "Calculation of MoM interaction integrals in highly conductive media," IEEE Trans. Antennas Propag., vol. 60, no. 2, pp. 930-940, Feb. 2012.

[28] K. Cools, F. P. Andriulli, F. Olyslager, and E. Michielssen, "Improving the MFIE's accuracy by using a mixed discretization," in Antennas and Propagation Society International Symposium, 2009. APSURSI '09. IEEE, Jun. 2009, pp. 1-4.

[29] S. Yan, J.-M. Jin, and Z. Nie, "Improving the accuracy of the secondkind Fredholm integral equations by using the Buffa-Christiansen functions," IEEE Trans. Antennas Propag., vol. 59, no. 4, pp. 1299-1310, Apr. 2011. 